\title{
Microdischarges for use as microthrusters: modelling and scaling
}

\author{
Ramesh A Arakoni $^{1,4}$, J J Ewing ${ }^{2}$ and Mark J Kushner ${ }^{3,5}$ \\ ${ }^{1}$ University of Illinois, Department of Aerospace Engineering, Urbana, IL 61801, USA \\ ${ }^{2}$ Ewing Technology Associates, Bellevue, WA 98006, USA \\ ${ }^{3}$ Iowa State University, Department of Electrical and Computer Engineering, Ames, IA 50011, USA \\ E-mail: ramesh.a.arakoni@intel.com,jjewingta@aol.com and mjk@iastate.edu
}

Received 23 December 2007, in final form 11 March 2008

Published 25 April 2008

Online at stacks.iop.org/JPhysD/41/105208

\begin{abstract}
Microsatellites with masses of tens of kilograms require only hundreds of micronewtons of thrust for station keeping and attitude control. Microdischarges (MDs) offer a compact way of generating such thrusts without using complex propulsion systems. In this paper, results from computational investigations of MDs sustained in Ar with tens of Torr back pressure are discussed with emphasis on conversion of discharge power into gas heating which can then be expanded in a nozzle to generate thrust. Typical cylindrical geometries have diameters of a few hundred micrometres and lengths of a few millimetres. We found that the gas temperature can exceed $1000 \mathrm{~K}$ for power densities of tens of kilowatts per cubic centimetre at back (upstream) pressures of tens of Torr. The nozzle length and location of the discharge in the MD channel are important from a gas dynamics viewpoint and so influence the incremental thrust (above that of the cold flow). Confining the discharge in the nozzle typically increases the peak gas temperature and the flow velocity, thereby potentially improving the performance of a MD used as a microthruster.
\end{abstract}

\section{Introduction}

Small satellites with masses of a few to tens of kilograms are being developed for phased-array radars and satellite networks [1]. Apart from the advantages of scalability and robustness, these systems also have a reduced risk of mission failure due to the redundancy available with a large fleet of microspacecraft. Efficient, noncontaminating and inexpensive thrusters are required for these small spacecraft to enable attitude and orbit control to increase their operational lifespan [2]. A variety of electrostatic and electromagnetic thrusters, such as Hall thrusters and pulsed plasma thrusters (PPTs), are now used in satellites for attitude control orbit manoeuvres, station keeping and de-orbiting [3-5]. Depending on size and mission, these microsatellites require millinewton to a few newtons of thrust, which in turn requires tens to hundreds of watts to operate. Scaling these devices down in power and size to suit the needs of microsatellites is a continuing challenge. For example, Hall thrusters make

${ }^{4}$ Present address: Intel, Inc., RA3-254, 250 NW 229th Avenue, Hillsboro, OR 97124, USA.

5 Author to whom any correspondence should be addressed. use of static magnetic fields to increase the residence times of electrons and the required strength of the magnetic fields scales inversely with the characteristic length. Microdischarge (MD) devices are also being developed as thrusters for microsatellites.

MDs based on cathode boundary layer [6] or hollowcathode excitation $[7,8]$ leverage $p d$ (pressure $\times$ distance) scaling to operate as dc discharges at pressures of hundreds of Torr to $1 \mathrm{~atm}$ and characteristic dimensions of tens to hundreds of micrometres. They are being investigated for possible use as, for example, excimer lighting sources [6], hydrogen production [7,8] and diamond deposition [9]. Radio frequency (rf) excited MDs are also being studied for use in lighting and surface modification [10-12]. For example, Eden et al [13] demonstrated MD sources operating at $5-20 \mathrm{kHz}$ using rare gas and $\mathrm{Ar} / \mathrm{N}_{2}$ mixtures in large-arrays of up to 40000 pixels.

MDs have also recently been investigated for use as microthrusters (MTs). Horisawa and Kimura [14] studied the feasibility of $2-20 \mathrm{~W}$ dc plasma jets as MTs using both gaseous $\left(\mathrm{N}_{2}\right)$ and solid propellants (Teflon). They generated millinewtons of thrust at $\mathrm{N}_{2}$ flow rates of a few milligrams per second (about $100 \mathrm{sccm}$ ) and found that 
increasing anode-cathode separation improved the thrust efficiency (incremental thrust per unit power). Slough et al [15] investigated the feasibility of dc MDs (300 $\mu$ m aperture at back pressures of $10 \mathrm{~s}$ Torr in Ar consuming 1-5 W) as thrusters. They measured a specific impulse $\left(I_{\mathrm{sp}}\right.$, defined as thrust per unit mass flow divided by gravity on earth's surface) of up to $220 \mathrm{~s}$ for $1 \mathrm{mg} \mathrm{s}^{-1}(30 \mathrm{sccm})$ of Ar resulting in a total thrust of $2.2 \mathrm{mN}$.

Kothnur and Raja [16] computationally investigated MDs (200 $\mu \mathrm{m}$ diameter) in 300-1000 Torr He and found them to operate in an abnormal glow regime. They also predicted high gas temperatures $(>1000 \mathrm{~K})$ and high plasma densities $\left(>10^{14} \mathrm{~cm}^{-3}\right)$. Takao and Ono [17] computationally investigated a miniature electro-thermal thruster using microwave excitation in Ar. They estimated a total thrust of $4.3 \mathrm{mN}$ at $3 \mathrm{~W}$. They also conducted preliminary experiments and obtained a steady discharge at powers as low as $1 \mathrm{~W}$.

MDs sustained in small volumes and high power densities (many to tens of kilowatt per cubic centimetre) result in significant gas heating (gas temperature $T_{\mathrm{g}}>1000 \mathrm{~K}$ ). The change in gas temperature occurs over small distances $(100 \mu \mathrm{m})$ leading to steep thermal gradients. These gradients produce a change in the velocity of the gas thereby generating an incremental change in thrust $(\Delta F)$ above that produced by expansion of a cold gas flow. In general, $\Delta F$ is a function of the power deposition, flow rate and upstream (or back) gas pressure. As such, the placement of the electrodes and the shape of the nozzle impact the location of the discharge and gas heating, and so affect the flow properties.

In this paper, we discuss results from a computational investigation of the properties of MDs configured for MT applications. In actual thrusters operating in a space environment, the downstream pressure is at or near vacuum, leading to supersonic and rarefied gas flows. Due to the complexities involving the transition from subsonic to supersonic and finally rarified flows, we imposed pressure boundary conditions where the flow remains continuum and flow does not transition to supersonic. As such, the intent of this work is to investigate the scaling of power transfer from electrical discharges to flowing gases in MD devices compatible with operation as MTs in spacecraft-like environments, though not necessarily operating in that regime. We expect the scaling laws we discuss for transfer of discharge power to gas flows in MDs will provide insights regardless of their outflow conditions.

The consequences of discharge configurations, power, pressure and flow rates on thrust are discussed. For example, for a cylindrically symmetric discharge sustained in Ar with diameter $600 \mu \mathrm{m}$ and electrode separation of $210 \mu \mathrm{m}$ (cathode located upstream in the gas flow) we predict $\Delta F=100$ $150 \mu \mathrm{N}$ for power depositions of $0.5-2 \mathrm{~W}$. (The upstream pressure of 50 Torr exhausts into 40 Torr.) The specific thrust (thrust per unit power deposition) ranged from 0.2 to $0.07 \mathrm{~N} \mathrm{~kW}^{-1}$ which is commensurate with electro-thermal propulsion devices.

The model used in this investigation is described in section 2 , followed by a discussion of results for sandwich-type
MDs (ring electrodes separated by a dielectric) in section 3. In section 4 we discuss results for simulations of MDs with the electrodes offset along the flow direction. Our concluding remarks are in section 5 .

\section{Description of model}

nonPDPSIM, a plasma hydrodynamics model, was used to investigate discharge enhanced flow properties in configurations compatible with the use of MDs as MTs. The model is described in detail in $[18,19]$, and so will be only briefly discussed here. nonPDPSIM consists of a plasma transport module coupled to a fluid dynamics module. Continuity equations for charged species, surface charges and Poisson's equation for the electric potential are implicitly integrated in time using Newton iterations and sparse matrix techniques on an unstructured mesh. The flux of charged species required in the transport equations are calculated using a modification of the Scharfetter-Gummel formulation [20]. Updates of potential and densities of charged species are followed by updates of the electron temperature $T_{\mathrm{e}}$ by solving its conservation equation using a successive-over-relaxation technique.

Electron transport coefficients and electron impact rate coefficients in the bulk plasma (where the reduced electric field $(E / N)$ is relatively low) are obtained from electron energy distributions (EEDs) calculated using a two-term spherical harmonic expansion of Boltzmann's equation [21]. The EEDs of beam-like secondary electrons emitted from the cathode and accelerated through the cathode fall are calculated using an electron Monte Carlo simulation. The rates of electron impact reactions are calculated by convolving cross-sections with the EEDs.

The neutral fluid flow is resolved by solving the compressible Navier-Stokes equations on the same unstructured mesh as the plasma calculations. The bulk fluid density $(\rho)$, momentum $(\rho \vec{V})$ and gas temperature $\left(T_{\mathrm{g}}\right)$ are simultaneously and implicitly solved by direct time integration. Momentum and energy transfer from charged particles to neutral species are also included in these conservation equations. The densities of individual neutral species are obtained by solving their own continuity equation using the bulk gas velocity $(\vec{V})$ and individual diffusion speeds while taking into account the heavyparticle reactions and electron impact collisions. The temperatures of solid materials were also solved for by extending the solution of the energy equation into materials while retaining only the thermal conduction term. In these cases, the outer boundary of the computational domain was held at $300 \mathrm{~K}$.

Secondary electron emission from surfaces is included both by ion impact and by thermal emission. A secondary electron emission coefficient of $\gamma=0.15$ was used for ion impact. Thermionic emission was assumed to be electric field enhanced:

$$
j_{\mathrm{e}}=A T_{\mathrm{S}}^{2} \exp \left(\frac{-\left(\Phi_{\mathrm{W}}-\left(q^{3} E / \varepsilon_{0}\right)^{1 / 2}\right)}{k T_{\mathrm{S}}}\right),
$$

where $\Phi_{\mathrm{W}}$ is the work function, $T_{\mathrm{S}}$ is the surface temperature, $A$ is the Dushman constant $\left(120 \mathrm{~A} \mathrm{~cm}^{-2} \mathrm{~K}^{-2}\right)$ and $E$ is the electric field at the surface. 
Metal surfaces are interfaced to an external circuit. The power deposition expended in the gas (not including any resistive circuit elements) is specified. The current flowing into the metal surfaces and the impedance of the circuit are used to determine the voltages on the metal surfaces. The power supply voltage is then periodically adjusted to deposit the specified power in the plasma.

The electron density $\left(n_{\mathrm{e}}\right)$ and ion density $\left(N_{\mathrm{i}}\right)$ are initialized with small values $\left(10^{9}-10^{11} \mathrm{~cm}^{-3}\right)$ having Gaussian distributions of width $100 \mu \mathrm{m}$ at the centre of the MD channel or adjacent to the cathode. The density and location of the initial seed of electrons were varied. Using lower values and alternate locations did not significantly affect the steady state plasma characteristics. The velocity and density of the gas at the inlet are calculated based on the specified flow rate and the upstream pressure. $T_{\mathrm{g}}$ is initialized to $300 \mathrm{~K}$ throughout the plasma domain. The outlets are sufficiently far away from the discharge region ( $>10$ times the diameter of the nozzle) that the axial gradients of flow quantities are approximately zero. The outlet boundary condition is obtained by specifying the downstream pressure and conserving mass flow through the system. Operationally, the model is first run without simulating the plasma in order to establish the cold gas flow operating conditions. Once this steady state is achieved, the voltage is turned on and the plasma equations solved. The species in the model are e, $\operatorname{Ar}, \operatorname{Ar}(4 s), \operatorname{Ar}(4 p), \mathrm{Ar}^{+}, \mathrm{Ar}_{2}^{*}$ and $\mathrm{Ar}_{2}^{+}$. The reaction mechanism is the same as discussed in [22].

The incremental thrust produced by the discharge, $\Delta F$, and the incremental thrust per unit power, $\Delta T_{\mathrm{s}}$, are defined as

$$
\begin{aligned}
\Delta F & =\left[\int_{0}^{R}\left(\rho v^{2}(r)+p(r)\right)(2 \pi r) \mathrm{d} r\right]_{d} \\
& -\left[\int_{0}^{R}\left(\rho v^{2}(r)+p(r)\right) 2 \pi r \mathrm{~d} r\right]_{0},
\end{aligned}
$$

$\Delta T_{\mathrm{s}}=\frac{\Delta F}{P}$,

where $P$ is the power, $\rho$ is the neutral gas density, $v(r)$ is the axial speed at radius $r, p(r)$ is the pressure and the integration is carried out over the exit plane of the nozzle. The subscripts $d$ and 0 represent the powered and cold gas flow, respectively. $\Delta T_{\mathrm{S}}$ is a measure of efficiency.

In the majority of cases discussed here, no-slip boundary conditions were used. For the lowest pressures and smallest devices, the Knudsen number $(K n)$ begins to approach 0.1 and so the no-slip boundary conditions are suspect [23]. Tests were performed of otherwise identical cases where either a no-slip or slip-flow boundary condition was used. For the latter, the gas speed at and parallel to the wall, $V_{\mathrm{w}}$, is given by

$$
V_{\mathrm{w}}=\lambda \frac{2-\sigma}{\sigma} \frac{\partial v}{\partial y}
$$

where $\lambda$ is the mean free path, $\sigma$ is the transverse momentum accommodation coefficient $(\sim 1)$, and $\partial v / \partial y$ is the gradient of the parallel velocity component in the perpendicular direction. In the worst case, we found differences in our thrust calculations of 2-3\% when comparing no slip and slip-flow boundary conditions.
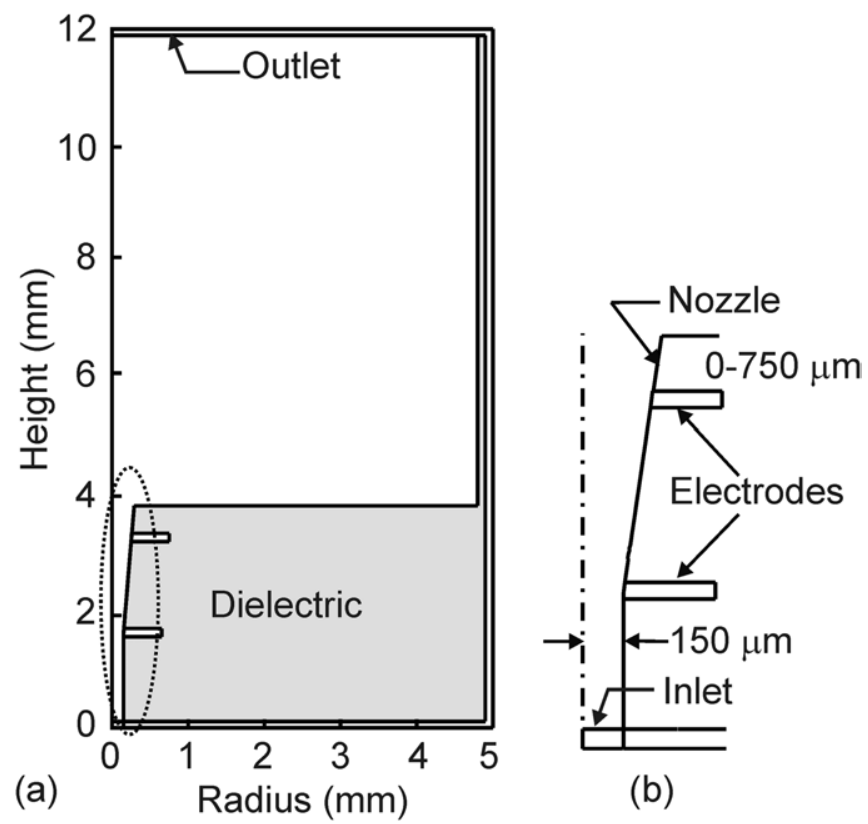

(b)

Figure 1. Schematic of the sandwich MD. (a) Full computational domain and $(b)$ enlargement of the discharge region.

\section{Sandwich MDs}

The sandwich MD configuration used in this investigation is shown in figure 1. The plasma channel has a diameter of $300 \mu \mathrm{m}$ and the nozzle divergence angle is $3.8^{\circ}$. The diameter of the nozzle at the exit is $500 \mu \mathrm{m}$ at the top of the downstream electrode. The thickness of the dielectric on top of the downstream electrode was varied from 0 to $750 \mu \mathrm{m}$. The metal electrodes are $125 \mu \mathrm{m}$ thick and the cathode is connected to the power supply through a $10 \mathrm{k} \Omega$ ballast resistor. The outer boundaries are electrically grounded and a layer of dielectric insulates the plasma from the boundary on the outer radius. This latter condition was for computational convenience to shield the ground plane.

For the base case, the inlet (back) Ar gas pressure is 30 Torr and the outlet pressure is 10 Torr. As such, there is a pressure gradient through the channel even with cold gas flow (no power deposition) which in turn produces cold gas flow thrust. The gas flow rate was varied from 8 to $30 \mathrm{sccm}$ $\left(\sim 0.25-0.89 \mathrm{mg} \mathrm{s}^{-1}\right)$ and the power deposition was varied from 0.25 to $1 \mathrm{~W}$.

The plasma potential, electron temperature $\left(T_{\mathrm{e}}\right)$ and ionization sources by bulk and beam electrons are shown in figure 2 for 30 Torr, $30 \mathrm{sccm}$ and $0.5 \mathrm{~W}$. The anode is upstream and the cathode is downstream. There is no dielectric on top of the cathode. The electron density $\left(n_{\mathrm{e}}\right)$, power deposition and density of $\mathrm{Ar}^{*}$ are shown in figure 3 . The gas speed, $\mathrm{Ar}$ density and gas temperature $T_{\mathrm{g}}$ are shown in figure 4 . The cathode voltage required to dissipate $0.5 \mathrm{~W}$ is $-256 \mathrm{~V}$.

The electrical conductivity of the channel is high enough that the anode potential is pulled well into the cylindrical channel creating a hollow-cathode-like configuration of electric potential. In the resulting cathode fall, the potential changes by nearly $240 \mathrm{~V}$ over $\approx 100 \mu \mathrm{m}$ producing an electric field in excess of $24 \mathrm{kV} \mathrm{cm}^{-1}$ and an $E / N$ (electric field/gas 
(a)

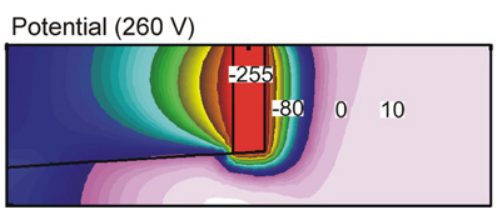

(b)

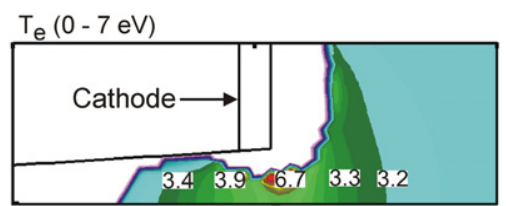

(c)

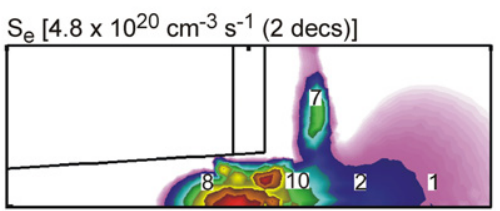

Beam $\left[4.8 \times 10^{20} \mathrm{~cm}^{-3} \mathrm{~s}^{-1}(2\right.$ decs $\left.)\right]$

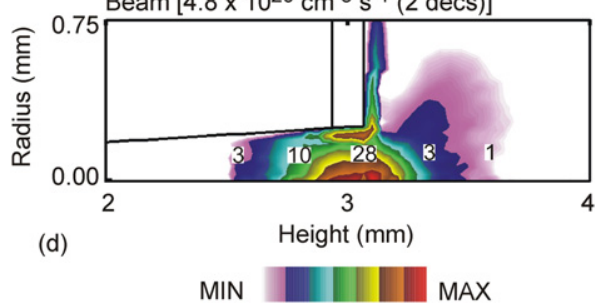

Figure 2. Plasma properties in the vicinity of the cathode for Ar, $0.5 \mathrm{~W}$ and $30 \mathrm{sccm}$ at 30 Torr inlet pressure. (a) plasma potential, $(b)$ electron temperature (where $n_{\mathrm{e}}>10^{-3}$ of peak value), $(c)$ ionization due to bulk electrons and $(d)$ ionization by beam electrons. The ionization sources are plotted on a 2-decade log scale with labels being percentages of the maximum value. Labels for $T_{\mathrm{e}}$ and potential are actual values.

number density) of $>4 \times 10^{-14} \mathrm{~V} \mathrm{~cm}^{2} . T_{\mathrm{e}}$ in the pre-sheath leading to the cathode is $6-7 \mathrm{eV}$ (results are only shown in figure 2 for volumes where $n_{\mathrm{e}}$ is greater than $10^{-3}$ of its maximum) whereas in the bulk plasma $T_{\mathrm{e}} \approx 4 \mathrm{eV}$. Ionization sources by electron impact have similar contributions from bulk electrons and from the beam electrons emitted from the cathode, both peaking at $5 \times 10^{20} \mathrm{~cm}^{-3} \mathrm{~s}^{-1}$. The ionization sources are largest inside the nozzle because the neutral gas and electron densities are highest there and there is a hollowcathode effect for secondary electrons emitted from the inside surfaces of the cathode.

Downstream of the cathode, the ionization sources are lower and more diffusive due to the lower gas density. The secondary electrons from the top cathode surface (facing the outlet) are accelerated in a lower electric field (due to the thicker sheath thickness) in a direction of lower gas density and so undergo fewer collisions per unit volume. The lack of a hollow-cathode effect on the front surface also reduces the rate of ionization. $T_{\mathrm{e}}$ is a few $\mathrm{eV}$ downstream of the cathode due to the lower electric field and so these electrons produce fewer ionizations. Power deposition has two contributions, from bulk electrons and from secondary electrons accelerated by the cathode fall, the latter contributing $<10 \%$ of the total in this case. A significant fraction $(\approx 30 \%)$ of the power is dissipated outside of the nozzle and so any gas heating produced by this power does not contribute to thrust.

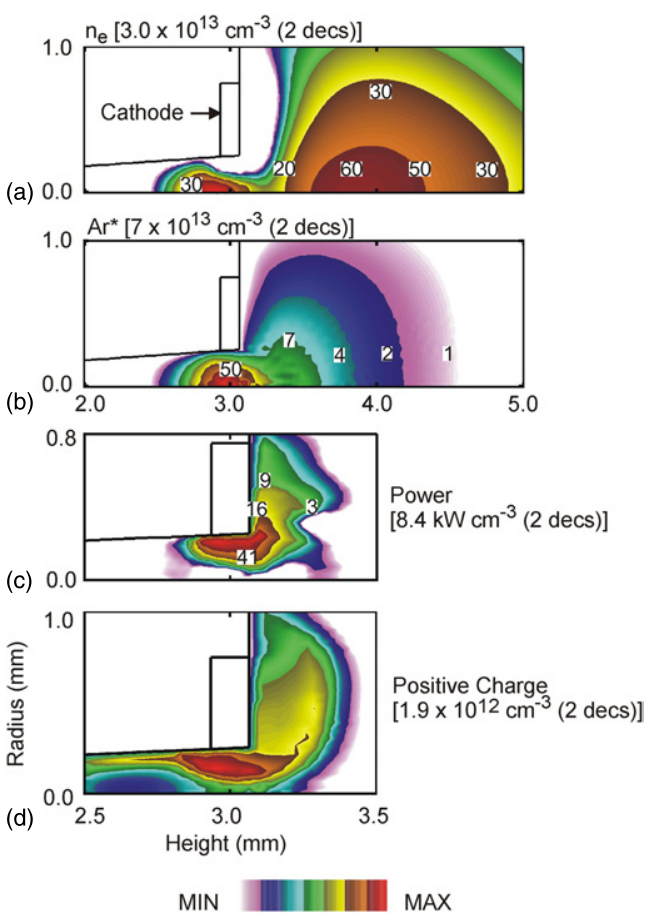

Figure 3. Plasma properties near the cathode for $\mathrm{Ar}, 0.5 \mathrm{~W}$ and $30 \mathrm{sccm}$ at 30 Torr inlet pressure. (a) Electron density, (b) Ar* density, $(c)$ power deposition and $(d)$ net positive charge density. All plots are on a 2-decade log scale and labels are percentages of maximum values. The electron density has two peaks where power deposition is maximum and downstream where losses are lower.

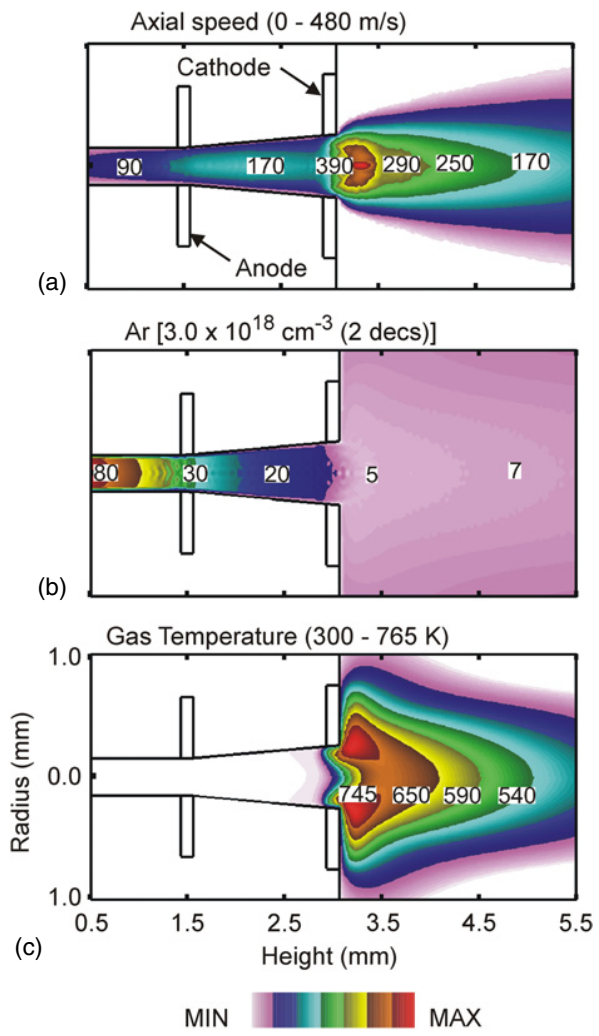

Figure 4. Neutral flow properties for $\mathrm{Ar} 0.5 \mathrm{~W}$ and $30 \mathrm{sccm}$ at 30 Torr inlet pressure. (a) Axial speed, (b) Ar density and (c) $T_{\mathrm{g}}$. Labels for the Ar density are percentages of the maximum value. The peak gas temperature is geometrically isolated from the maximum in flow speed. 
The electron density has two peaks. The first is on the axis inside the nozzle $\left(3.0 \times 10^{13} \mathrm{~cm}^{-3}\right)$ adjacent to the cathode. Here the ionization by beam electrons is large by virtue of a hollow-cathode effect and the gas density is large enough to slow beam electrons. The excited state density is also high $\left(7 \times 10^{13} \mathrm{~cm}^{-3}\right)$ so that multi-step ionization is rapid, by both beam and bulk electrons. The second peak in electron density is downstream of the cathode $\left(2 \times 10^{13} \mathrm{~cm}^{-3}\right)$. Outside the nozzle, the effective diffusion length is longer thereby decreasing rates of loss by diffusion so that the lower ionization rates produce commensurate electron densities to that in the nozzle. Although $T_{\mathrm{e}}$ is lower in this regions $(3 \mathrm{eV})$ ionization by Penning collisions $\left(3 \times 10^{17} \mathrm{~cm}^{-3} \mathrm{~s}^{-1}\right)$ produces about $10 \%$ of the total and helps sustain the plasma. The pinching of the electron density between the upstream and downstream peaks is due to the extension of the cathode fall from the vertex of the cathode, whose large negative potential excludes bulk electrons. The peak of electron density downstream is augmented by the advective flow of the gas which entrains the ions and, through ambipolar forces, drags along electrons.

The Ar* density is the sum of the densities of $\operatorname{Ar}(4 s)$ and $\operatorname{Ar}(4 p)$ which, due to the short lifetime of $\operatorname{Ar}(4 p)$, is dominated by $\operatorname{Ar}(4 \mathrm{~s})$. The $\operatorname{Ar}(4 \mathrm{~s})$ is consumed by Penning reactions (with a lifetime of $\approx 10 \mu \mathrm{s}$ ), by 3 -body reactions with $\mathrm{Ar}$ atoms producing $\mathrm{Ar}_{2}^{*}$ (lifetime of $\approx 90 \mu \mathrm{s}$ ) and, downstream where $T_{\mathrm{e}}$ is small, by super-elastic electron collisions (lifetime of $\approx 20 \mu \mathrm{s}$ ). These processes dominate over losses due to diffusion. The fractional power dissipated by radiation is typically $<0.5 \%$.

Gas heating in large part results from Joule heating by ion collisions (e.g. elastic collisions and symmetric charge exchange) and secondarily by elastic collisions with electrons. Net momentum transfer from the electric field through ions and electrons is only important in nonneutral regions such as the cathode fall. For this geometry, this momentum transfer opposes the direction of net thrust though the fractional contribution is small $(<1 \%)$. Typically, the momentum transfer from ion-neutral collisions is important only when the degree of ionization is large $\left(>10^{-3}\right)$.

Energy transfer processes increase $T_{\mathrm{g}}$ from $300 \mathrm{~K}$ at the inlet to $765 \mathrm{~K}$ adjacent to the cathode where the drift of ions back towards the cathode produces a maximum in Joule heating. The plume of hot gas downstream results from the advective flow of the heated gas. As the gas cools further downstream, the resulting expansion leads to an increase in neutral gas speed from a cold thrust value of $310 \mathrm{~m} \mathrm{~s}^{-1}$ to a peak value of $480 \mathrm{~m} \mathrm{~s}^{-1}$. Due to the high surface-to-volume ratio of this device and the fact that the walls have a large thermal mass, the heat transfer to the walls is significant. For example, Williams et al [24] predicted that the thermal loss to the walls to be $\approx 60-70 \%$ of the input power at pressures of 45 60 Torr in an electro-thermal MT having similar dimensions. In devices where the temperature of the walls significantly increases, the heating is not necessarily all bad as the energy lost to the walls is recouped by the incoming gas which gets pre-heated as it enters the discharge region.

The neutral gas density is highest near the inlet and decreases steadily as the gas flows through the nozzle. During this expansion, the Ar density decreases from $2.4 \times 10^{18} \mathrm{~cm}^{-3}$ at the inlet to $1.5 \times 10^{17} \mathrm{~cm}^{-3} 0.5 \mathrm{~mm}$ downstream of the cathode. The reduction in gas density at the outlet is due to a combination of the initial condition (a pressure gradient across the device producing cold gas thrust) and gas heating by the discharge. With a constant mass flow rate, the change in gas speed due to heating by the discharge produces an increase in thrust over the cold gas value.

The consequences of momentum transfer from directed ion (or electron) fluxes, sometimes referred to the ion wind, has potentially important implications to MTs. There is only net momentum transfer from the plasma to the neutral flow in regions where there is a net charge density. On the scale of the dynamic range plotted for the electron density in figure 3(a), the ion density is essentially the same. The net positive charge density is shown separately in figure $3(d)$. The charge density has a maximum of $1.8 \times 10^{12} \mathrm{~cm}^{-3}$ in the plasma-side of the cathode fall where the electric field is at most a few hundreds of volts per centimetre. The resulting ion wind imparts a drift speed of a few to many tens of centimetres per second whereas the axial flow speed is many thousands of centimetres per second. So for these conditions, the ion wind does not play a major role.

The consequences of power deposition $(0.2-1 \mathrm{~W})$ on plasma properties (cathode fall voltage, $n_{\mathrm{e}}$ and $T_{\mathrm{e}}$ ) and flow speed are shown in figure 5. $T_{\mathrm{e}}$ is measured at the centre of the channel in the bulk plasma near the cathode (height $=3 \mathrm{~mm}$ ) and $n_{\mathrm{e}}$ is the maximum value. The cathode voltage and $n_{\mathrm{e}}$ increase nearly linearly with power indicating the MD is operating in an abnormal glow regime, as Kothnur and Raja found in their device [16]. $T_{\mathrm{e}}$ decreases by $0.45 \mathrm{eV}(4.25-$ $3.8 \mathrm{eV}$ ) with power deposition over this range. This decrease is in part attributable to an increase in Ar* densities thereby increasing the rate of multi-step ionization and an increase in the contributions to ionization by beam electrons due to the higher cathode voltage, both of which allow the self-sustaining $T_{\mathrm{e}}$ to decrease. As the cathode potential increases with power (becomes more negative) and the gas rarefies due to more gas heating, $E / N$ in the pre-sheath increases. The larger $E / N$ and larger ion density with power increases the ion flux to the walls and to the cathode in particular. The ions recombine on surfaces and dissipate an energy at least equal to its ionization potential $(16 \mathrm{eV})$ and at maximum would be the entire cathode potential. Since the mean free path for charge exchange is a few to $10 \mu \mathrm{m}$, the ions strike the cathode with a small fraction of the cathode fall voltage. This is a power loss which might otherwise be used in gas heating. Over the range of powers investigated, this loss is not more than a few per cent of the total.

The increase in gas temperature above the inlet value of $300 \mathrm{~K},(\Delta T)$, and the gas speed at the throat of the nozzle are shown in figure $5(c)$. These values are the maximum in each case and occur $\approx 100-150 \mu \mathrm{m}$ downstream of the cathode. $\Delta T$ and the increase in speed are nearly linear with power deposition at lower powers and begin to saturate near $1 \mathrm{~W}$. The maximum $\Delta T$ is $\approx 700 \mathrm{~K}$ and the speed increases from 415 to $610 \mathrm{~m} \mathrm{~s}^{-1}$ as the power is increased from 0.0 to $1.0 \mathrm{~W}$. The saturation in $\Delta T$ and speed is not unexpected. At higher 
(a)
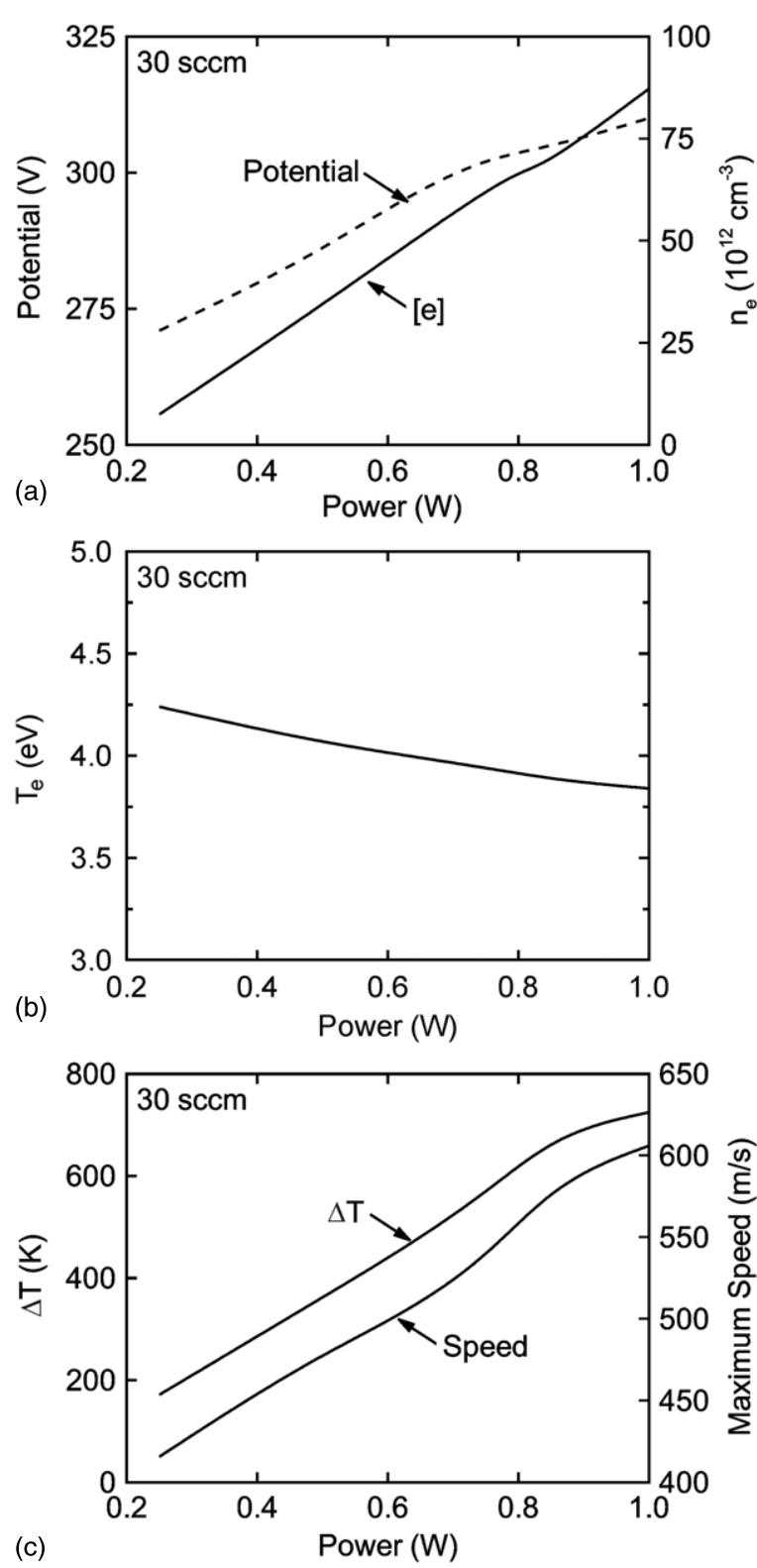

Figure 5. Plasma and flow properties while varying power for 30 Torr and $30 \mathrm{sccm}$ of Ar. (a) Plasma potential and $n_{\mathrm{e}},(b) T_{\mathrm{e}}$ in the bulk plasma and (c) $\Delta T$ and maximum axial speed. The maximum $\Delta T$ and axial speed occur 100-150 $\mu \mathrm{m}$ downstream of the nozzle exit plane.

power deposition and greater rarefaction, the mean free paths of secondary electrons are longer and so beam ionization and ion production occurs over a larger volume. Since gas heating is dominated by ion Joule heating which has a diminishing specific rate, $\Delta T$ saturates.

The consequences of flow rate and power deposition on $\Delta T$ and gas speed $150 \mu \mathrm{m}$ downstream of the nozzle exit are shown in figure 6 . The residence time of gas between the electrodes decreases with increasing flow rate, thereby decreasing the specific energy deposition per atom for a given power. For example, from 7.5 to $30 \mathrm{sccm}$, the specific energy deposition decreases from 1.8 to $0.44 \mathrm{eV} /$ atom. As a result $\Delta T$ decreases with increasing flow rate. The speeds at the nozzle exit increase with power for a given flow rate (more gas heating)
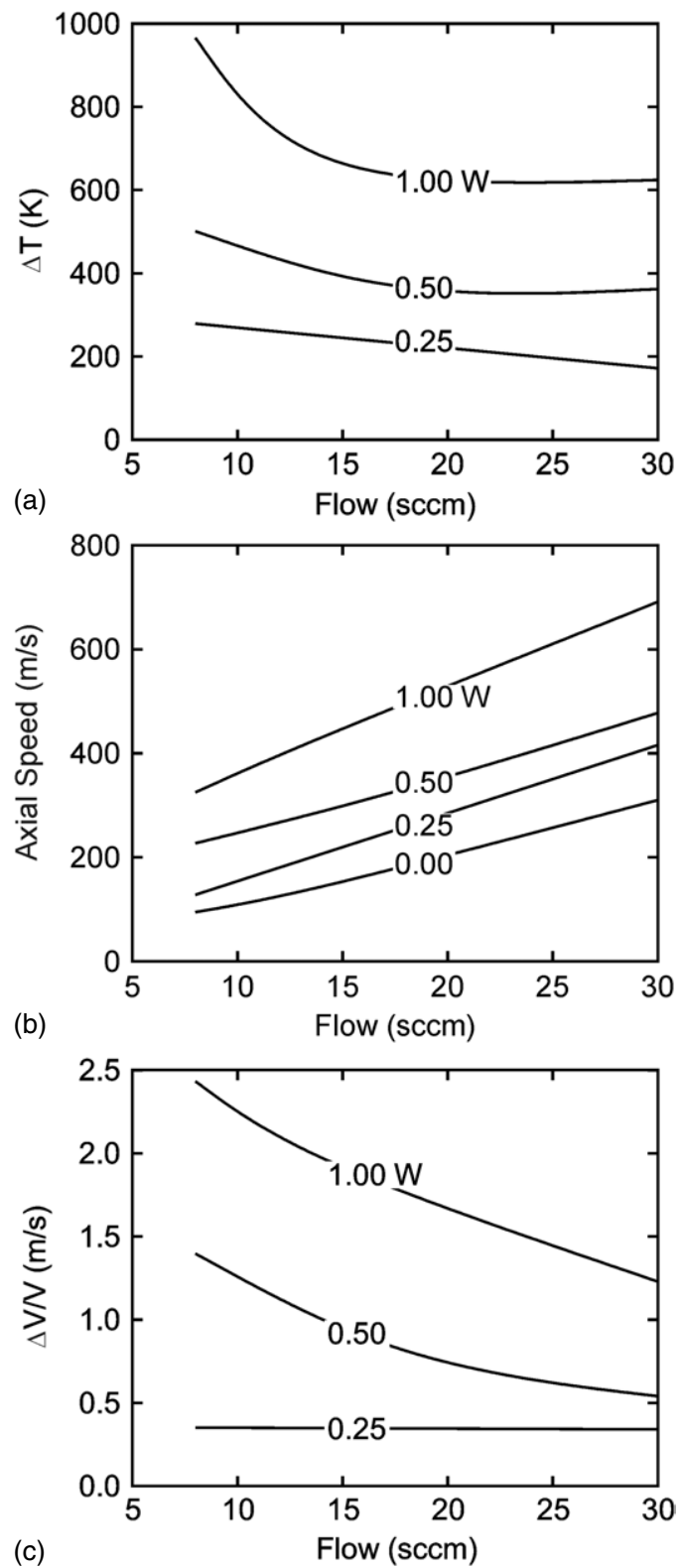

Figure 6. Plasma and flow properties as a function of flow rate for different powers at 30 Torr inlet pressure. (a) $\Delta T$, (b) axial speed and $(c)$ relative incremental velocity $(\Delta V / V)$. The MD is more efficient at low flow rates and high power where the initial kinetic energy of the flow is smaller.

and increase with flow rate for a given power (increase in the cold gas contribution to thrust). To isolate the contributions of the cold gas flow, the increase in axial speed relative to the cold gas value is shown in figure 6(c). The MD is most efficient at transferring power to the gas in terms of increase in speed $(\Delta V)$ at low flow rates and high powers where $\Delta V$ is larger compared with the cold gas speed $V$. This is not unexpected because at low flow velocities, the kinetic energy in the flow, $\rho V^{2} / 2$, is small compared with the power from the discharge, and so the discharge has a larger influence on $\Delta V$.

The consequences of nozzle design on the discharge and flow properties were investigated by adding a layer of dielectric insulation on top (downstream) of the cathode and varying its thickness up to $750 \mu \mathrm{m}$ (see figure 1.) The plasma properties 
(a)

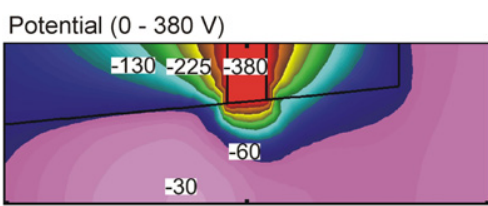

Power $\left[60 \mathrm{~kW} \mathrm{~cm}^{-3}\right.$ (2 decs)]

(b)

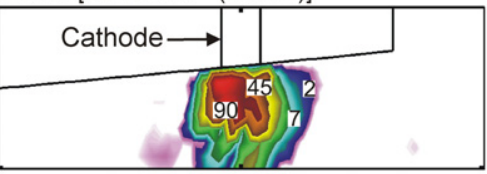

$\mathrm{S}_{\mathrm{e}}\left[5.1 \times 10^{21} \mathrm{~cm}^{-3} \mathrm{~s}^{-1}(2\right.$ decs $\left.)\right]$

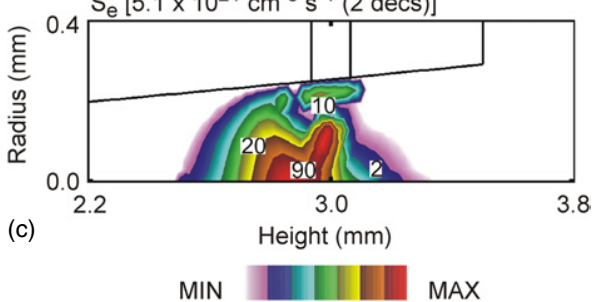

MIN

Figure 7. Plasma properties for the sandwich MD near the cathode with a $500 \mu \mathrm{m}$ dielectric layer on top of the cathode. (a) Electric potential, $(b)$ power density and $(c)$ ionization sources. Power deposition and ionization sources are concentrated in the interior of the nozzle. The contours for power and ionization are plotted on a 2-decade log scale with labels being a percentage of the maximum value.

for a MD with a $500 \mu \mathrm{m}$ thick dielectric are shown in figure 7 for $1 \mathrm{~W}$ and $30 \mathrm{sccm}$ Ar flow with an inlet pressure of 30 Torr. Power deposition is concentrated into a smaller volume near the cathode producing a higher peak value $\left(60 \mathrm{~kW} \mathrm{~cm}^{-3}\right)$ than with the top surface of the cathode being exposed. With roughly the same current, secondary electrons are emitted from only the inner surface of the cathode and so the current density of ionizing beam electrons is higher. As a result, the ionization sources are restricted to a small volume spanning $400 \mu \mathrm{m}$ upstream of the cathode, with nearly no ionization sources downstream. The curvature of the cathode fall results from there being a higher plasma density upstream producing a smaller sheath thickness. This curvature orients trajectories of beam electrons upstream in spite of the slant of the face of the cathode which faces the other direction.

The consequences of dielectric thickness on top of the cathode on the electron density are shown in figure 8 for $30 \mathrm{sccm}$ Ar at 30 Torr back pressure and $1 \mathrm{~W}$. When the cathode is exposed and the top surface emits secondary electrons, the peak electron density is $8.7 \times 10^{13} \mathrm{~cm}^{-3}$ (figure $8(a)$ ) and there is a secondary maximum downstream of $2.4 \times 10^{13} \mathrm{~cm}^{-3}$. With the addition of the dielectric on top of the cathode (figures $8(c)$ and $(d)$ ), large ionization sources are restricted to the volume between the electrodes. Higher maximum values of $n_{\mathrm{e}}$ of $2.4 \times 10^{14} \mathrm{~cm}^{-3}$ are produced which are not sensitive functions of dielectric thickness. The electrons downstream of the nozzle diffuse in an almost electric field free region with little additional ionization and so their density is low $\left(10^{11} \mathrm{~cm}^{-3}\right)$.

The shift in location of the plasma when covering the top dielectric is due, in part, to negative charging of the dielectric walls downstream of the cathode which confines the plasma. However, the majority of the effect is due to current and

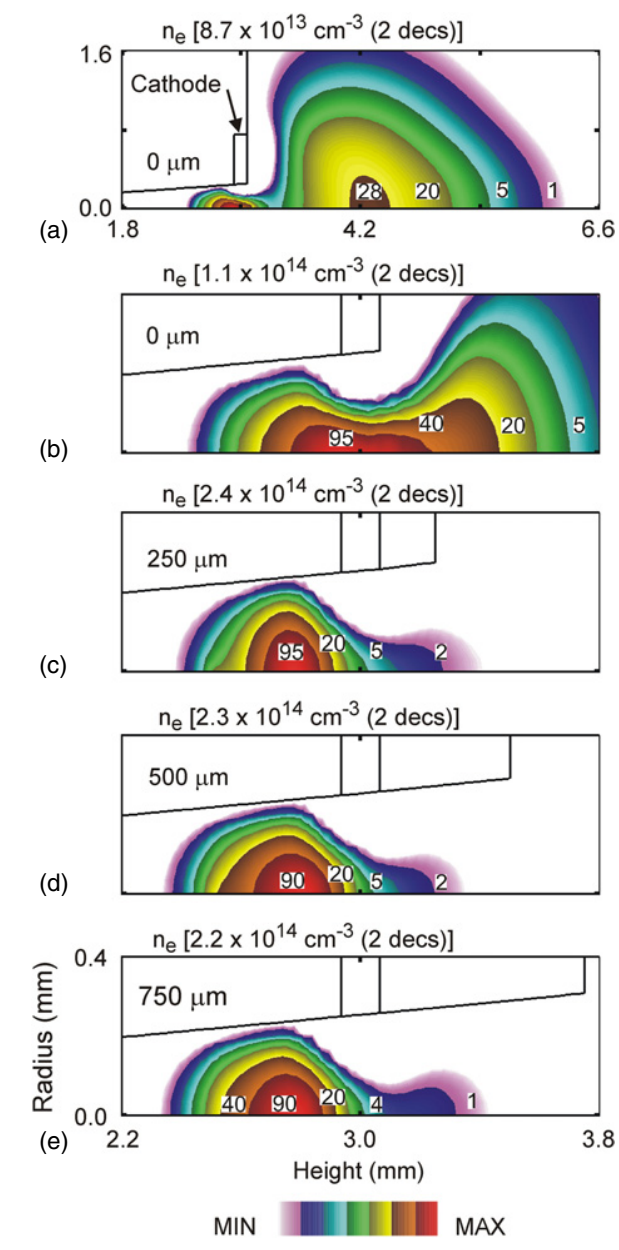

Figure 8. Electron density for Ar, 30 Torr, $30 \mathrm{sccm}$ and $1 \mathrm{~W}$ while varying the thickness of the dielectric on top of the cathode: $(a)$ No dielectric, $(b)$ no dielectric and without secondary electron emission from the top surface of the cathode, (c) $250 \mu \mathrm{m}$ thick dielectric, (d) $500 \mu \mathrm{m}$ and $(e) 750 \mu \mathrm{m}$. The contours are plotted on a 2 -decade log scale with labels being percentage of maximum values. The dielectric cap confines the plasma to inside the nozzle producing a higher specific power deposition.

secondary electrons being emitted only from only the innerwalls of the cathode. This higher current density produces a larger specific ionization source. As such, the increase in peak $n_{\mathrm{e}}$ is a consequence of the maximum power deposition increasing from $14 \mathrm{~kW} \mathrm{~cm}^{-3}$ with the exposed cathode to $60 \mathrm{~kW} \mathrm{~cm}^{-3}$ with the covered cathode, tempered by increased rates of loss by recombination and diffusion with the more confined plasma.

Changing the thickness of the dielectric did not significantly affect the plasma properties once the top surface of the cathode was covered and secondary electron emission from the top surface was curtailed. To demonstrate this effect, the secondary electron emission coefficient on the top surface of the exposed cathode (no dielectric layer) was set to zero so that only the inner surfaces of the cathode emitted secondary electrons. In this case (figure $8(b)$ ), the plasma is not as confined as with a dielectric cap due to the lack of negative charging of that top surface, although the peak $n_{\mathrm{e}}$ increases from $8.7 \times 10^{13}$ to $1.1 \times 10^{14} \mathrm{~cm}^{-3}$. With only secondary emission from the inner surface of the cathode, the peak power 

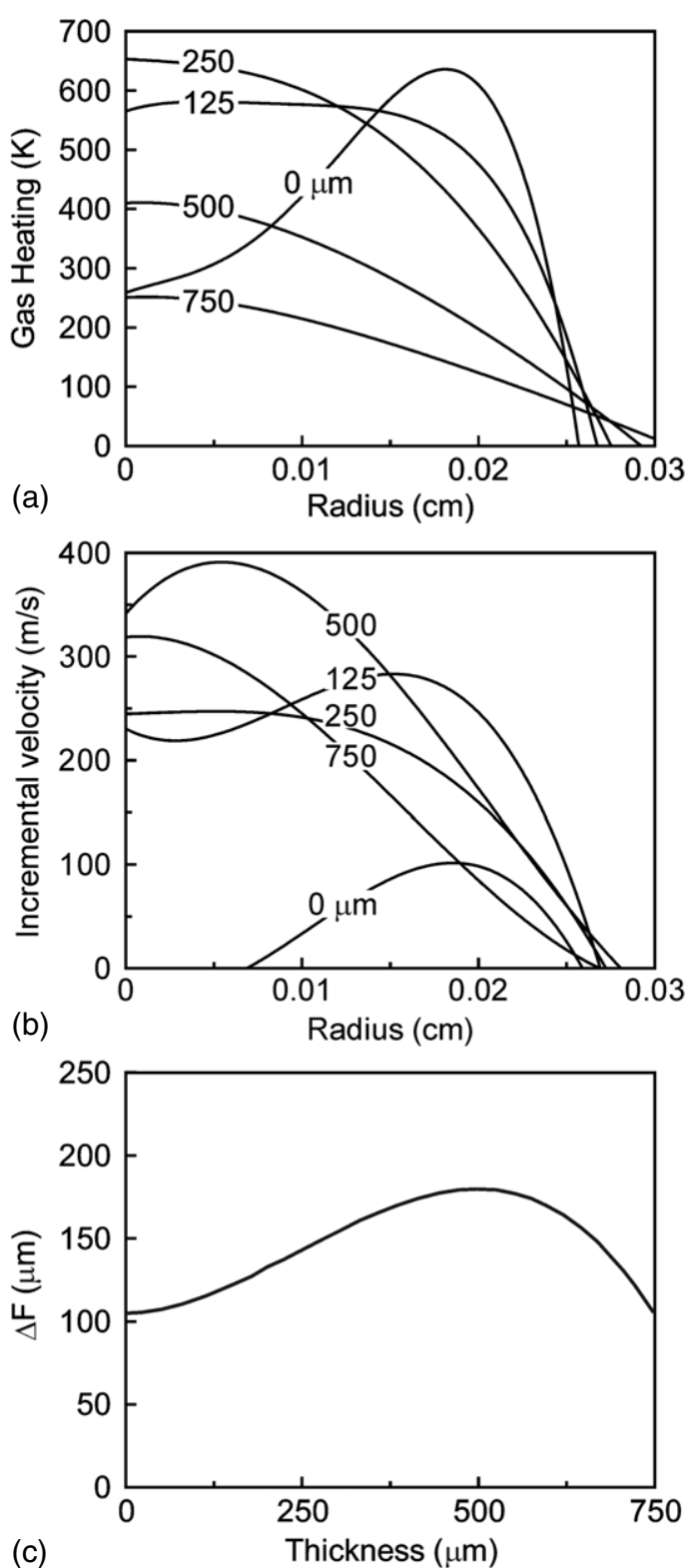

Figure 9. Neutral flow properties as a function of dielectric thickness and radius at the exit of the nozzle for 30 Torr, $30 \mathrm{sccm}$ and $1 \mathrm{~W}$. (a) $\Delta T$, (b) $\Delta V$ (above the cold flow) and (c) $\Delta F$. The optimum dielectric cap maximizes specific power deposition while minimizes heat transfer to walls.

density increases a factor of two compared with when the entire cathode emits secondary electrons. The secondary, downstream peak in electron density is removed without secondary emission from the top surface of the cathode, indicating that the ionization produced by beam electrons from the top surface plays some role in sustaining the secondary peak. Managing the location of secondary electron emission is important to the confinement of the plasma.

The increase in specific power deposition resulting from confinement of the plasma by the dielectric covering the cathode results in higher gas temperatures and gas exit speeds on axis. For example, the increase in gas temperature, $\Delta T$, the increase in gas speed relative to cold gas flow, $\Delta V$, and the incremental thrust, $\Delta F$ are shown in figure 9 at the nozzle exit plane for different dielectric thicknesses. In the absence of the

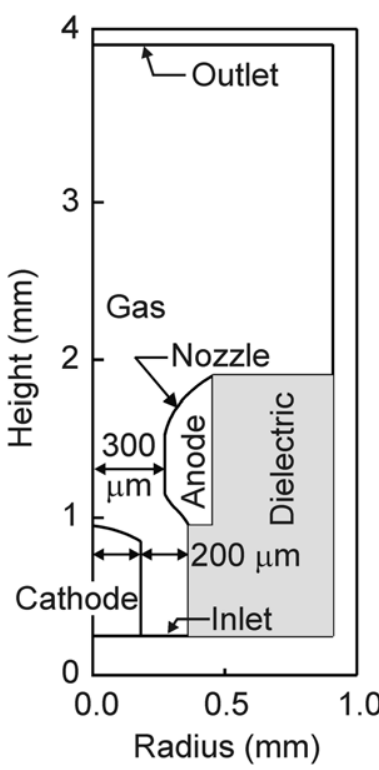

Figure 10. Schematic of the MAT having a cylindrical cathode on axis and the anode part of the side wall. Gas enters through the annular volume around the cathode.

dielectric, the gas heats nearest the cathode and cools as the gas exits the discharge. $\Delta T$ and $\Delta V$ are highest at large radius in the boundary layer and so do not translate efficiently into thrust. By confining the discharge with the dielectric over-layer, the peak power deposition and gas heating are higher midstream, thereby producing higher local values of $\Delta T$ and $\Delta V$. A relatively thin dielectric cap, $125-250 \mu \mathrm{m}$, optimizes $\Delta T$ to as large as $650 \mathrm{~K}$ in the centre of the channel whereas $\Delta V$ optimizes with an over-layer of about $500 \mu \mathrm{m}$ at $400 \mathrm{~m} \mathrm{~s}^{-1}$. With thicker caps, the heated gas travels a longer distance beyond the location of peak power deposition before reaching the exit of the nozzle, and has more opportunity to cool thereby lowering exit speeds. This effect is likely exaggerated here as the wall temperatures are close to $300 \mathrm{~K}$.

The incremental speed, $\Delta V$, is an indication of the increase in recoverable thrust due to the discharge. For this particular geometry and power, the incremental thrust is highest when the dielectric cap on the cathode is $500 \mu \mathrm{m}$. If the nozzle length is too short, the thermal energy in the gas is diffused in the downstream volume and so is underutilized. If the nozzle is too long, then losses to the walls can be large. For example, as an extreme case of nozzle length, the polarities of the anode and cathode were reversed so that the discharge took place deep inside the nozzle. In this case, the $\Delta V$ at the nozzle exit was nearly zero as the gas had cooled before reaching the exit plane.

\section{Annular MDs}

In the sandwich-type MDs discussed above, power deposition is close to the side-walls of the nozzle which can lead to loss of power by heat conduction. To position the discharge at the centre of the flow and so reduce these losses, the annular MD shown in figure 10 was investigated. In this geometry (referred to as a microarc thruster, MAT), a cylindrical cathode (400 $\mu$ m diameter) is on the axis of the flow channel, producing 
(a)

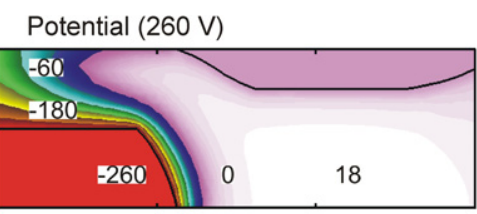

Beam $\left[1.93 \times 10^{21} \mathrm{~cm}^{-3} \mathrm{~s}^{-1}(2\right.$ decs $\left.)\right]$

(b)

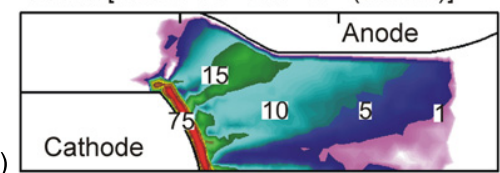

(c)

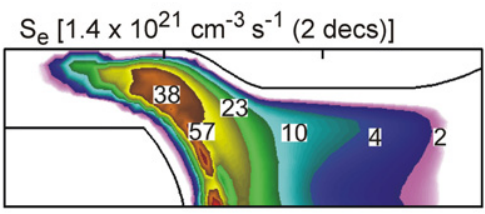

(d)
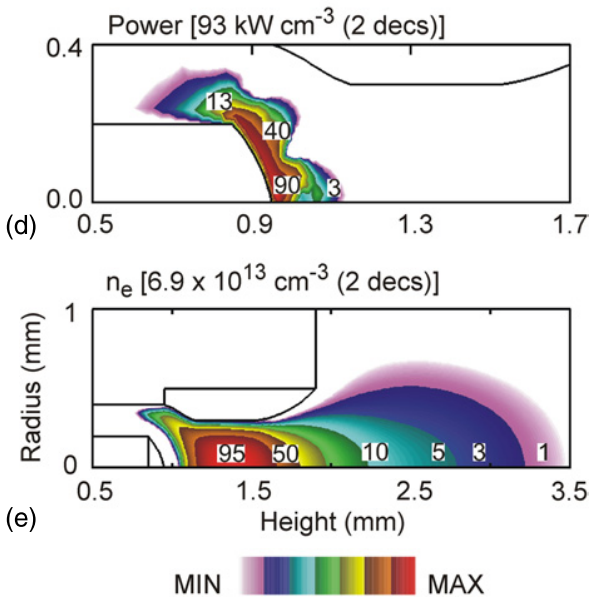

Figure 11. Plasma properties for the MAT near the cathode for $1 \mathrm{~W}$ and $25 \mathrm{sccm}$ of Ar at 25 Torr inlet pressure. (a) Electric potential, (b) ionization by beam electrons, (c) ionization by bulk electrons, $(d)$ power deposition and $(e) n_{\mathrm{e}}$. All contours except the potential are on a 2-decade log scale with the labels being percentages of the maximum values.

an annular inlet flow of $200 \mu \mathrm{m}$ around the cathode. The anode is part of the nozzle walls and the separation between the electrodes is $\approx 210 \mu \mathrm{m}$. The cathode is experimentally observed to heat to nearly melting [25] so computationally the initial temperature of the cathode was $800 \mathrm{~K}$, a value lower than that expected for gas temperatures near the cathode. The boundary and initial conditions are otherwise the same as those described above.

The plasma characteristics for the MAT are shown in figure 11 for the base case of 25 Torr exhausting into 20 Torr, $1 \mathrm{~W}$ and $25 \mathrm{sccm}$ of Ar. The plasma spreads over the entire face of the nozzle and diffuses $>2 \mathrm{~mm}$ downstream whereas significant power deposition is confined to 150 $200 \mu \mathrm{m}$ downstream of the cathode. With the cathode fall facing the direction of gas flow, secondary electrons emitted from cathode produce ionizations along the flow direction. Ionization by beam electrons near the cathode fall is about 1.5 times that by bulk electrons. However, bulk electrons also produce significant ionization upstream in the annular region. The peak $n_{\mathrm{e}}$ occurs in the centre of the discharge at the throat of the nozzle and extends downstream. A well-developed sheath near the cathode leads to a considerable net positive space

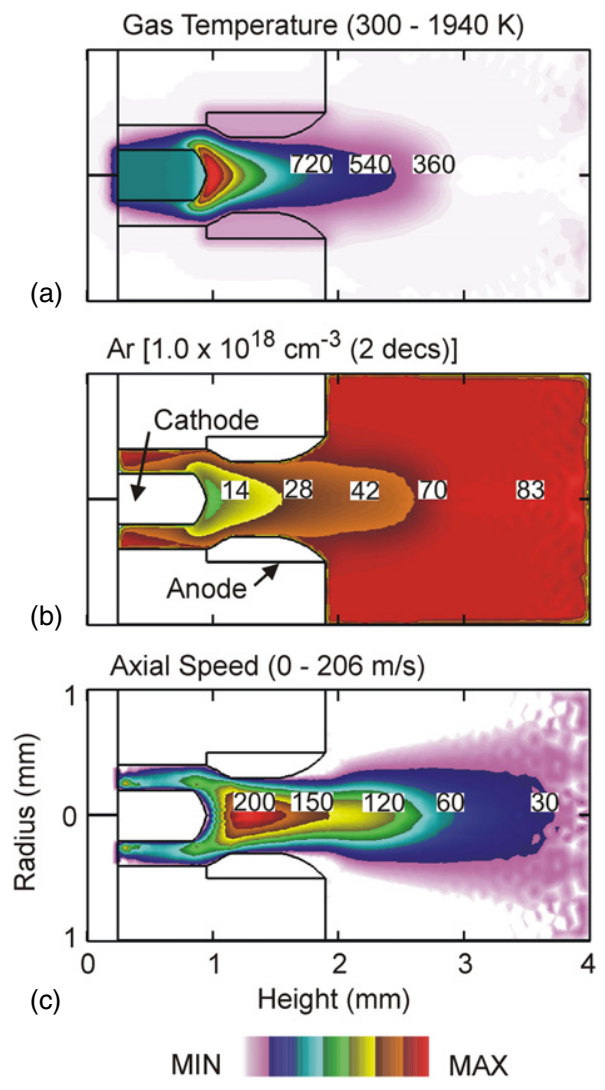

Figure 12. Flow properties for the MAT for $1 \mathrm{~W}$ and $25 \mathrm{sccm}$ of $\mathrm{Ar}$ at 25 Torr inlet pressure. (a) $T_{\mathrm{g}}$, (b) Ar density and (c) axial speed. The Ar density is plotted on a 2-decade log scale with labels being percentage of the maximum value. The maximum gas temperature occurs in a stagnation zone. The stippling in $(c)$ is a plotting artifact.

charge producing a net acceleration of the neutral gas by ion collisions opposite that of the flow. However, the absolute magnitude of the ion-drag acceleration caused by this space charge is small.

The neutral gas density, $T_{\mathrm{g}}$, and axial speed in the MAT are shown for the base case in figure 12. In this geometry, a stagnation point $(V=0)$ occurs at the tip of the cathode. As a result, the forced convection of gas away from this location is small and so the gas temperature is higher (1940 K) compared with the sandwich discharge. The conduction of heat through the cathode wire leads to preheating of gas as it flows around the cathode into the discharge in front of the cathode. The peak flow speed is $206 \mathrm{~m} \mathrm{~s}^{-1}$.

Gas heating produces rarefaction in the discharge and so influences the $E / N$ and ionization sources. For example, beam ionization sources and $n_{\mathrm{e}}$ are shown in figures 13 and 14 as a function of power for 25 Torr upstream pressure and $25 \mathrm{sccm}$ of Ar. With an increase in power, $T_{\mathrm{g}}$ increases and the gas density decreases due to rarefaction. The mean free path of secondary electrons increases and ionization by beam electrons extends further downstream of the cathode. Due to there being fewer collisions, more secondary electrons are directly collected by the anode before they are slowed in the gas. The end result is that at higher powers, the ionization region is larger, extends further downstream and is dominated by beam electrons. At lower powers, the ionization sources are diffusive and relatively concentrated in the narrow gap between 


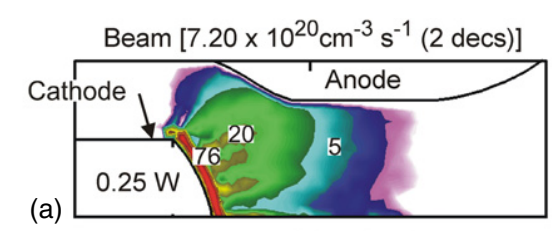

Beam $\left[1.35 \times 10^{21} \mathrm{~cm}^{-3} \mathrm{~s}^{-1}(2\right.$ decs $\left.)\right]$

(b)

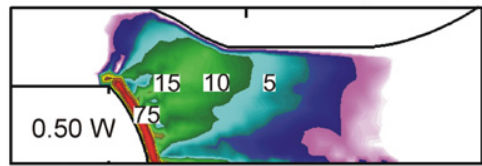

Beam $\left[1.88 \times 10^{21} \mathrm{~cm}^{-3} \mathrm{~s}^{-1}(2\right.$ decs $\left.)\right]$

(c)
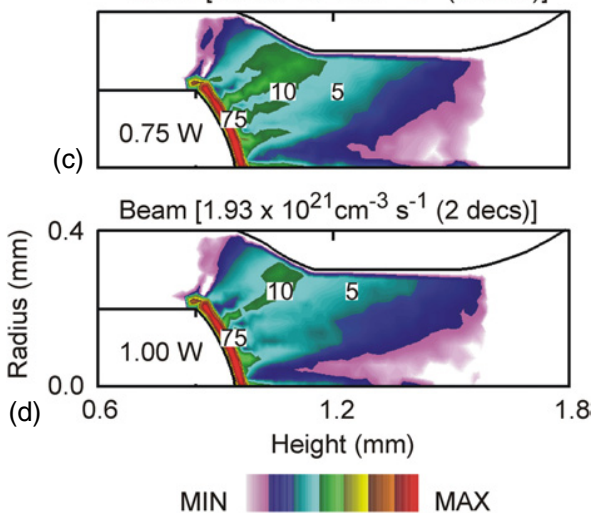

Figure 13. Beam ionization sources for the MAT while varying power for $25 \mathrm{sccm}$ Ar at 25 Torr. (a) $0.25 \mathrm{~W},(b) 0.5 \mathrm{~W}$, (c) $0.75 \mathrm{~W}$ and $(d) 1 \mathrm{~W}$. The contours are a 2 -decade log scale with labels being percentages of the maximum value. Increasing power extends ionization sources downstream.

(a)
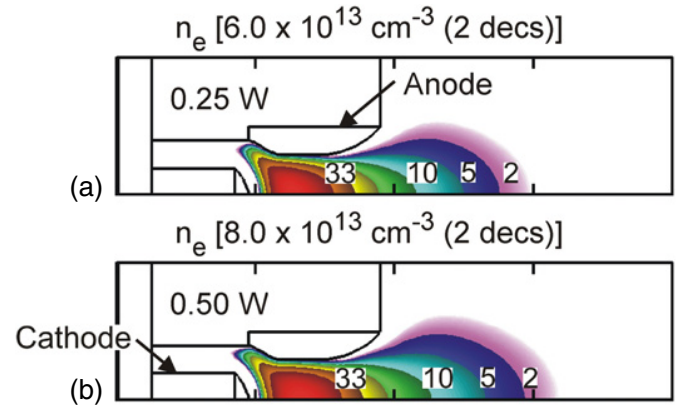

(b)

$$
\mathrm{n}_{\mathrm{e}}\left[8.7 \times 10^{13} \mathrm{~cm}^{-3}(2 \text { decs })\right]
$$

(c)
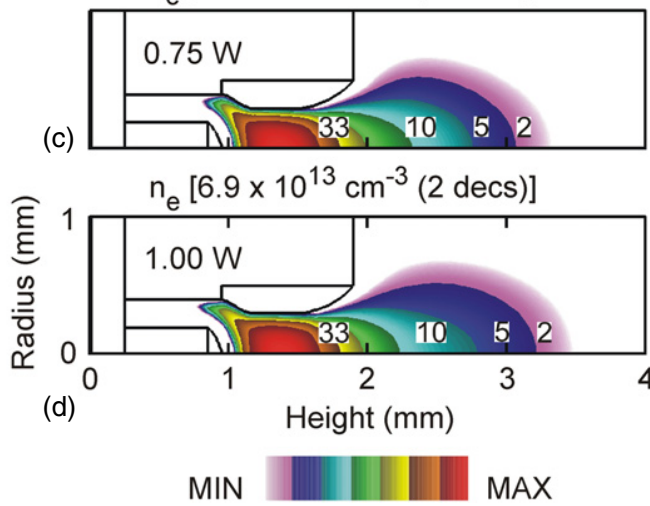

Figure 14. Electron density for the MAT while varying power for $25 \mathrm{sccm}$ Ar at 25 Torr. (a) $0.25 \mathrm{~W},(b) 0.5 \mathrm{~W},(c) 0.75 \mathrm{~W}$ and $(d) 1 \mathrm{~W}$. The contours are a 2 -decade log scale and labels are percentages of the maximum value. Electron density extends downstream with increasing power deposition.

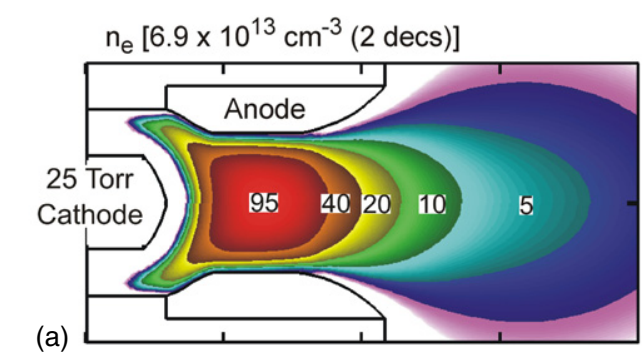

$\mathrm{n}_{\mathrm{e}}\left[5.0 \times 10^{14} \mathrm{~cm}^{-3}(2\right.$ decs $\left.)\right]$

(b)
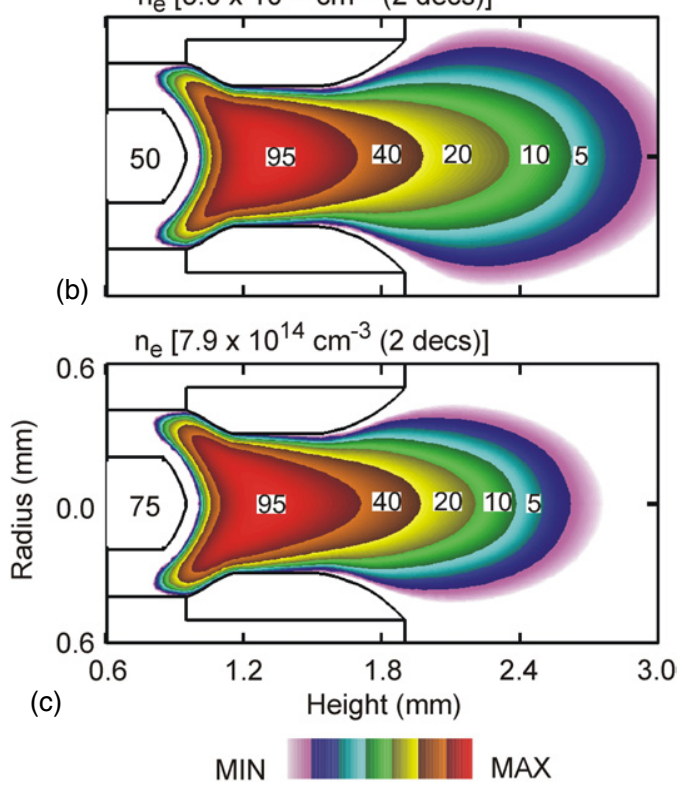

Figure 15. Electron density for the MAT while varying inlet pressure for $1 \mathrm{~W}$. (a) 25 Torr, (b) 50 Torr and (c) 75 Torr. The contours are a 2-decade log scale with labels being percentages of the maximum value. The plasma is restricted to a smaller volume at higher pressures producing higher electron densities.

the electrodes. The ionization sources due to bulk electrons behave similarly with power deposition. The end result is an extension of $n_{\mathrm{e}}$ downstream with increasing power deposition.

The consequences of upstream pressure (25-75 Torr) on $n_{\mathrm{e}}$ are shown in figure 15 for $1 \mathrm{~W}$. The inlet sccm is scaled with pressure to keep the cold gas residence time constant. The electron collision frequency increases with pressure, and so ionization sources and power deposition are confined to a smaller volume within the nozzle as the pressure increases. Consequently, $n_{\mathrm{e}}$ increases with pressure, from $6.9 \times 10^{13}$ to $7.9 \times 10^{14} \mathrm{~cm}^{-3}$ from 25 to 75 Torr.

The consequences of power deposition on the maximum values of $n_{\mathrm{e}}$ and $\Delta T$ are shown in figure 16 for different pressures in the MAT. $n_{\mathrm{e}}$ increases with pressure for a given power but weakly depends on power at low pressures. An increase in power deposition increases the ionization sources but does so over a larger volume. This occurs while the gas density decreases due to an increase in $T_{\mathrm{g}}$. The diffusion of electrons is more rapid at lower neutral densities with there being a lower specific power deposition, which leads to a reduction in the peak $n_{\mathrm{e}}$. The end results is that $n_{\mathrm{e}}$ may decrease with power in spite of there being a larger $\mathrm{eV}$ per atom deposited in the flow and so a larger $\Delta T$. The peak gas temperature occurs near the tip of the cathode where the 

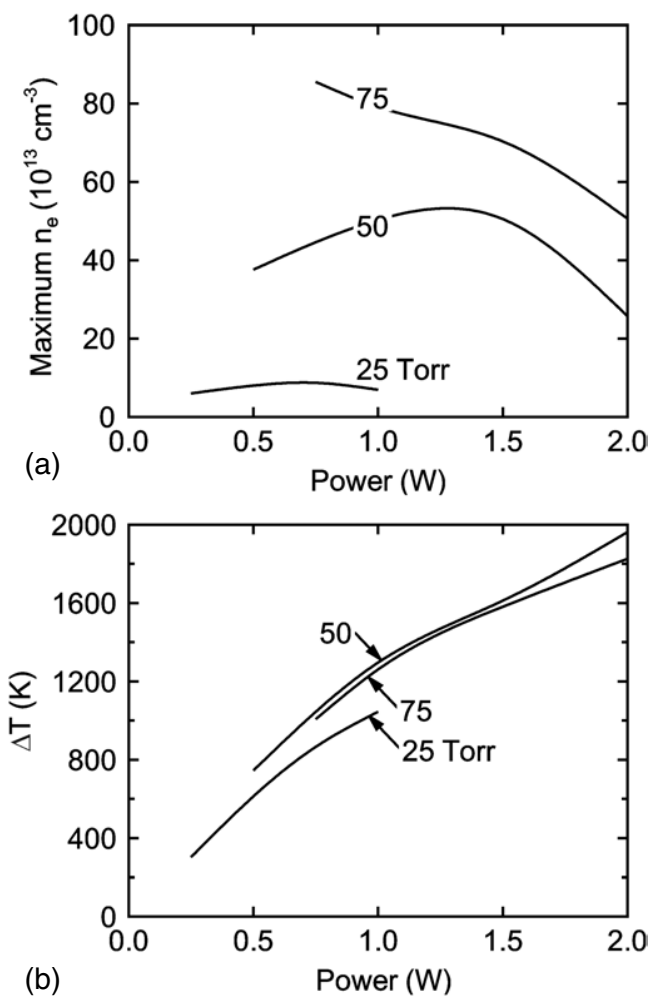

Figure 16. Plasma and flow properties as a function of power and pressure for the MAT. (a) Maximum electron density and (b) $\Delta T$. $T_{\mathrm{g}}$ increases with power but is a weak function of pressure.
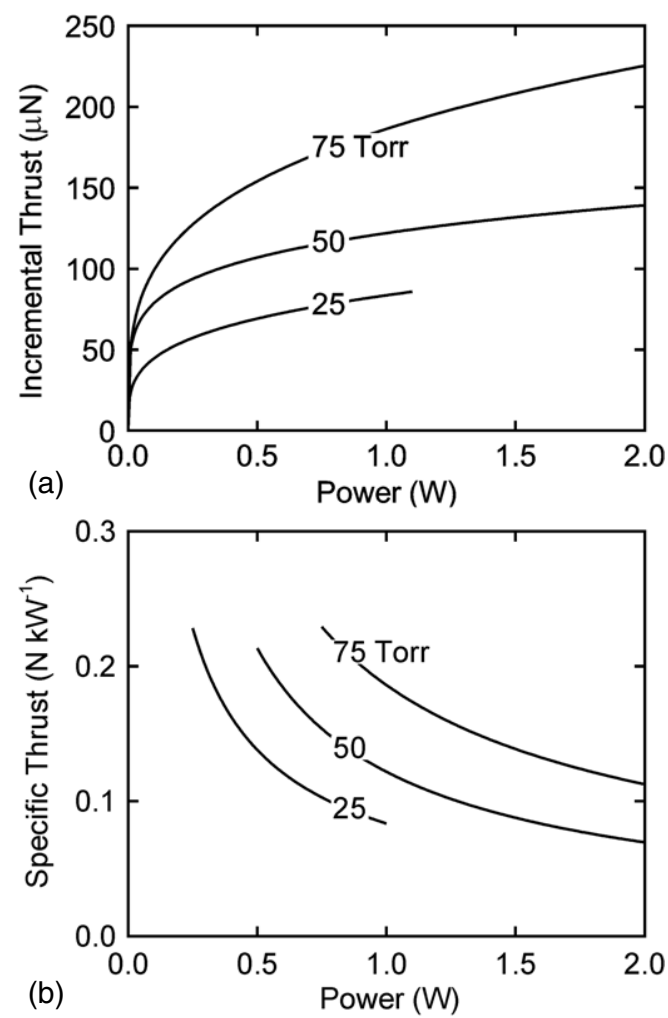

Figure 17. Thrust properties for the MAT as a function of power for different pressures. (a) Incremental thrust above cold gas flow $\Delta F$ and $(b)$ specific thrust $\Delta T_{\mathrm{S}}$. Although thrust increases with power and pressure, the efficiency (specific thrust) decreases.
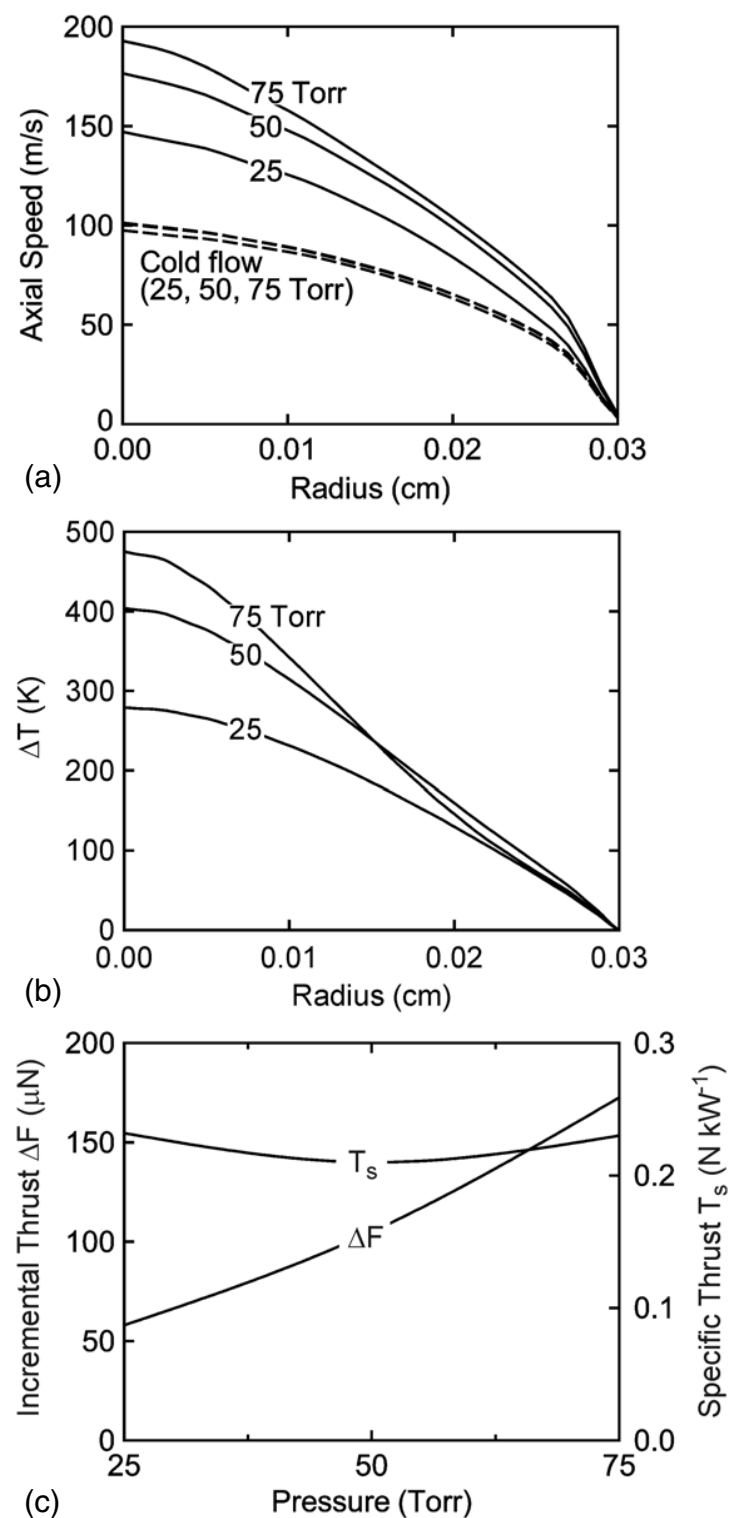

Figure 18. Neutral flow properties as a function of radius for the MAT while varying pressure. The residence time $(13 \mu \mathrm{s})$ and energy deposition per atom $(0.14 \mathrm{eV} /$ atom $)$ are constant. Flow values at 25 Torr are $25 \mathrm{sccm}$ and $0.25 \mathrm{~W}$. Flow rate and power are scaled with pressure. (a) Axial speed at nozzle exit (height $=1.9 \mathrm{~mm}$ ), (b) $\Delta T$, (c) $\Delta F$ and $\Delta T_{\mathrm{s}}$. Thrust increases with pressure at nearly constant efficiency.

power deposition is high, and the convection is low. The $\Delta T$ increases almost linearly with power deposition as expected, and has a weaker dependence on pressure.

The incremental thrust $\Delta F$ at the exit plane of the MAT and the specific thrust $\Delta T_{\mathrm{S}}$ as a function of power are shown in figure 17 for different pressures. $\Delta F$ increases to $225 \mu \mathrm{N}$ for 75 Torr and $1 \mathrm{~W} . \Delta F$ increases rapidly with small power and saturates at higher powers for all pressures. As a result, the specific thrust $\Delta T_{\mathrm{s}}$ decreases with power. The saturation with power is due to increases in power losses (conduction to a larger area of walls with a larger $\Delta T$ ), and to a smaller degree sheath losses and expansion of the plasma. However, for a given power, $\Delta T_{\mathrm{s}}$ is higher at higher pressures. 
Axial speed at the exit plane and increase in gas temperature $\Delta T$ as a function of radius, and incremental thrust $\Delta F$ and specific thrust $\Delta T_{\mathrm{s}}$ as a function of pressure are shown in figure 18 . The energy deposition per atom $(0.14 \mathrm{eV} /$ atom $)$ and cold gas residence time $(13 \mu \mathrm{s})$ were kept constant. Since the flow rate increases in proportion to pressure, the cold gas velocity profiles are essentially the same. $\Delta T$ is higher at higher pressures due to the confinement of the plasma in a smaller volume and so axial speeds increase with pressure. The incremental thrust $\Delta F$ increases almost linearly with pressure to reflect the increase in $\Delta T$. In contrast, $\Delta T_{\mathrm{s}}$ is almost a constant with pressure due to the $\mathrm{eV}$ per atom being constant.

\section{Concluding remarks}

Results from a computational investigation of cylindrically symmetric MDs were discussed with an emphasis on converting discharge power into thermal power for their possible use as MTs. In the MDs investigated, the plasma is sustained by ionization by both bulk and secondary electrons. The power deposition is typically concentrated near the cathode surface, leading to high power densities and high gas temperatures, and so geometries which optimize the overlap between gas flow and peak power deposition tend to maximize thrust. In general, discharges that were confined to be inside the nozzle produced higher gas heating and larger incremental thrusts ranging from 50 to $200 \mu \mathrm{N}$ for power depositions of $0.25-2 \mathrm{~W}$. $\Delta F$ generally scales sub-linearly with power for a given pressure, producing lower values of specific thrust, $\Delta T_{\mathrm{s}}$, due to rarefaction which reduces the specific power deposition.

\section{Acknowledgments}

This work was supported by the Air Force Office of Scientific Research, the National Science Foundation (CTS-0520368) and by Ewing Technology Associates.

\section{References}

[1] Janson S W, Helvajian H, Hansen W W and Lodmell J 1999 Batch-fabricated cw microthrusters for kilogram-class spacecraft Proc. 35th AIAA/ASME/SAE/ASEE Joint Propulsion Conf. Exhibit. (Los Angeles, CA) AIAA Paper 1999-2722

[2] Mueller J 2000 Thruster options for microspacecraft: a review and evaluation of state-of-the-art and emerging technologies Micropropulsion for Small Spacecraft ed M M Micci and A D Ketsdever (Reston, VA: AIAA)

[3] Martinez-Sanchez M and Pollard J E 1998 J. Propulsion Power 5688

[4] Burton R L and Turchi P J 1998 J. Propulsion Power 5716

[5] Jahn R G Physics of Electric Propulsion (New York: McGraw-Hill)

[6] Moselhy M and Schoenbach K H 2004 J. Appl. Phys. 951642

[7] Qiu H, Martus K, Lee W Y and Becker K 2004 Int. J. Mass Spectrom. 23319

[8] Hsu D D and Graves D B 2005 Plasma Chem. Plasma Proc. 251

[9] Sankaran R M and Giapis K P 2002 J. Appl. Phys. 922406

[10] Lauer J L et al 2004 J. Appl. Phys. 964539

[11] Kikuchi T, Hasegawa Y and Shirai H 2004 J. Phys. D: Appl. Phys. 371537

[12] Ichiki T, Taura R and Horiike Y 2004 J. Appl. Phys. 9535

[13] Eden J G, Park S J, Ostrom N P and Chen K F 2005 J. Phys. D: Appl. Phys. 381644

[14] Horisawa H and Kimura I 2001 Very low-power DC plasma-jet-microthrusters Proc. 37th AIAA/ASME/SAE/ASEE Joint Propulsion Conf. Exhibit. (Salt Lake City, UT) AIAA Paper 2001-3791

[15] Slough J, Andreason S, Ziemba T and Ewing J J 2005 Micro-discharge micro-thruster Proc. 41st AIAA/ASME/SAE/ASEE Joint Propulsion Conf. Exhibit. (Tucson, AZ) AIAA Paper 2005-4074

[16] Kothnur P S and Raja L L 2005 J. Appl. Phys. 97043305

[17] Takao Y and Ono K AIAA 2004-3621

[18] Kushner M J 2004 J. Appl. Phys. 95846

[19] Arakoni R A, Stafford D S, Babaeva N Y and Kushner M J 2005 J. Appl. Phys. 98073304

[20] Scharfetter D L and Gummel H K 1969 IEEE Trans. Electron Devices 1664

[21] Rockwood S D 1973 Phys. Rev. A 82348

[22] Bhoj A N and Kushner M J 2004 J. Phys. D: Appl. Phys. 372510

[23] Schaaf S A and Chambre P L 1961 Flow of Rarefied Gases (Princeton, NJ: Princeton University Press)

[24] Williams K L et al 2006 J. Micromech. Microeng. 161154

[25] Slough J and Ewing J J 2007 Microarcjet-microthruster for nanosat applications Proc. 43rd AIAA/ASME/SAE/ASEE Joint Propulsion Conf. Exhibit. (Cincinnati, OH) AIAA Paper 2007-5181 